\title{
Free Falling Szilard Engine, Entropic Forces and Landauer's principle; Revisiting the Principle of Equivalence
}

\author{
Edward Bormashenko ${ }^{1^{*}}$, and Michael Nosonovsky ${ }^{2,3}$
}

1 Department of Chemical Engineering, Biotechnology and Materials, Engineering Science Faculty, Ariel University, Ariel, 40700, Israel. Tel.: +972-074-7296863; edward@ariel.ac.il

2 X-BIO Institute, University of Tyumen, 6 Volodarskogo St, Tyumen, 625003, Russia. Tel. +7-345-259-7425;

3 Department of Mechanical Engineering, University of Wisconsin-Milwaukee, 3200 North Cramer St, Milwaukee, WI 53211, USA, Tel. +1-414-229-2816; nosonovs@uwm.edu

* Correspondence: edward@ariel.ac.il

\begin{abstract}
Gedanken experiments illustrating exemplifications of the Landauer principle in the free falling Einstein elevator are treated. Double-well simplest information system embedded into the free falling elevator is addressed. Infinitesimal horizontal force applied to the particle $m$ transfers it from position " 0 " to position " 1 ", emerging from the free falling double-well system confining mass $m$. When thermal noise is considered, the potential barrier of $k_{B} T$ should be surmounted for the erasing of one bit of information. Entropic forces arising in the free falling elevator are considered. The maximal change in the entropy of free-joint polymer chain attached to the free falling elevator is estimated as $\left|\Delta S_{\text {max }}\right| \cong k_{B}$, and it is remarkably independent of the mass attached to the chain and the parameters of the chain itself. Free falling minimal Szilard engine is treated. The informational re-interpretation of the minimal Szilard process is shaped as follows: the energy $k_{B} T \ln 2$ necessary for erasing of 1 bit of information is spent for lifting up mass, whatever, is the value of this mass. Appropriate choice of frames enables elimination of gravity in the considered system; however elimination of the thermal noise (dissipation processes) by the same procedure is impossible.
\end{abstract}

Keywords: Principle of equivalence; Landauer principle; Entropic forces; Information; Einstein free falling elevator.

\section{Introduction}

John Archibald Wheeler suggested that fundamentals of physics should be re-built on the informational groundings and assumed that the main notions of physics are deeply rooted in the "bit-based" scientific paradigm [1]. This global scientific program was extensively developed in the last decade. The informational re-shaping of thermodynamic of gaseous and condensed phases, exploiting the Shannon measure of information was addressed in refs. 2-6. Informational content of the molecular motion was treated in ref. 7. Information-based re-interpretation of mechanics was discussed in refs. 8-14. Information aspects of detecting particles were considered in ref. 15. Information approach to the general relativity was suggested in refs. 16-17.

The Landauer Principle, suggesting the thermodynamic equivalent of information, serves a natural basis for informational re-shaping of physics. The Landauer principle formulated in 1961 in ref. 18 and revisited thirty years later in ref. 19 states that the erasure of one bit of information requires a minimum energy consumption equal to $\boldsymbol{k}_{\boldsymbol{B}} \boldsymbol{T} \boldsymbol{l n} \mathbf{2}$, where $T$ is the temperature of a thermal reservoir used in the process and $\boldsymbol{k}_{\boldsymbol{B}}$ is Boltzmann's constant. Let us quote ref. 18 , it is plausible to anticipate, "that information processing is inevitably accompanied by a certain minimum amount of heat generation. In a general way this is not surprising. Computing, like all processes proceeding at a finite rate, must involve some dissipation ... the dissipation has a real function and is not just an unnecessary nuisance... note that a binary device must have at least one degree of freedom associated with the information. Classically a degree of freedom is associated with $\boldsymbol{k}_{\boldsymbol{B}} \boldsymbol{T}$ of thermal energy. Any switching signals passing between devices must therefore have this much energy to override the noise...An alternative way of anticipating our conclusions is to refer to the arguments by Brillouin and earlier authors, as summarized by Brillouin in his book, "Science and Information Theory," to the effect that the measurement process requires a dissipation of the order of $\boldsymbol{k}_{\boldsymbol{B}} \boldsymbol{T}^{\prime \prime}$. Rolf Landauer, 
also addressed in Ref. 18, the model of the particle enclosed in a bi-stable well, imitating the simplest logical cell. Rolf Landauer also applied the aforementioned principle to the transmission of information and re-worded it as follows: an amount of energy equal to $\boldsymbol{k}_{\boldsymbol{B}} \boldsymbol{T} \boldsymbol{l n} \mathbf{2}$ (where $\boldsymbol{k}_{\boldsymbol{B}} \boldsymbol{T}$ is the thermal noise per unit bandwidth) is needed to transmit a bit of information, and more if quantized channels are used with photon energies $\boldsymbol{h} \boldsymbol{v}>\boldsymbol{k}_{\boldsymbol{B}} \boldsymbol{T}$ [20]. Actually the Landauer principle, pertaining to the lower theoretical limit of energy consumption of computation, converted the information into physical value, thus, bridging between information of measurable physical values.

The derivation, meaning interpretation of the Landauer principle were subjected to the intensive scientific discussion [22]. Bennett in ref. 22, argued that the Landauer principle represents actually restatement of the Second Law of Thermodynamics, however it has considerable pedagogic and explanatory power, especially in the context of other influential ideas in nineteenth and twentieth century physics [22]. We present the information-based re-interpretation of the Einstein equivalence principle.

\section{Results and discussion.}

\subsection{The Einstein equivalence principle and the two-well Landauer information model.}

The Einstein equivalence principle is a basis of general relativity and the entire building of modern physics [2326]. The understanding of the Einstein equivalence principle of general relativity remains ambiguous [27]. According to the classical textbook by Landau and Lifshitz non-inertial frame is equivalent to a gravitational field [23]. According to Tolman the equivalence principle is formulated as follows: the effect of homogenous and stationary gravitational field may be always eliminated by the appropriate choice of the accelerated frames [24]. Landau and Lifshitz also stressed the fundamental difference between a "true gravity" and non-inertial frames: a true gravity always disappears far from gravitational masses, whereas pseudo-gravitational fields emerging in non-inertial frames remain finite in infinity [23].The useful discussion of the precise meaning and interpretation of the equivalence principle is supplied in ref. 27.

We adhere to the very tight interpretation of the Einstein equivalence principle, namely the possibility to eliminate the effects due to gravity by the appropriate choice of the accelerated frames, and suggest its information-based reinterpretation. Consider the bi-stable potential well placed in the free falling elevator, as shown in Figure 1. Such a well may be seen as an elastic frictionless bowl containing particle $m$. Let us arbitrarily label the particle $m$ located in the lefthand well as the ZERO state. When the particle is in the right-hand well, the device is in the ONE state, as suggested by Rolph Landauer in ref. 18. Thus, we combine the Einstein Gedanken free falling elevator with the simplest implementation of the binary logical device, introduced by Rolph Landauer [18-20]. Let us place particle $m$ in the well labeled "0", as depicted in Figure 1. It is easily seen that in the free falling elevator the energy barrier disappears, and an infinitesimal horizontal force $\overrightarrow{\boldsymbol{f}}$ applied to the particle transfers it from position "ZERO" to position "ONE" (again, friction is absent in the system). Indeed, force $\overrightarrow{\boldsymbol{f}}$ gives rise to the non-compensated vertical component of the elastic reaction force. This force will bring particle to the apex of the well and afterwards it will bring it to the position " 1 " state. 


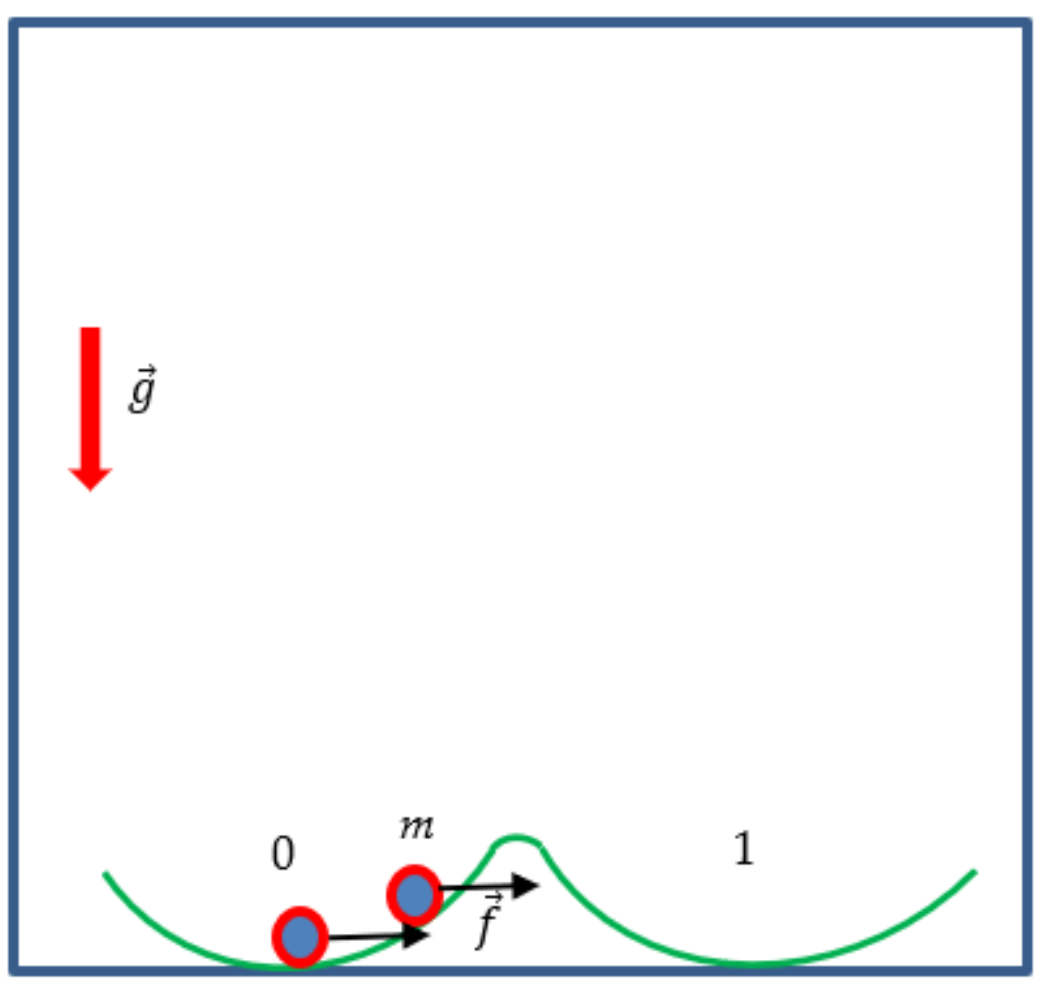

Figure 1. Bi-stable potential well placed within the free falling elevator, built of two wells labeled correspondingly " 0 ” and " 1 . Infinitizemal horizontal force $\vec{f}$ applied to the particle $m$ will transfer it from the well labeled " 0 " to the well labeled "1".

Now let us follow the reasoning suggested by Rolph Landauer in ref.18, when applied to the bi-stable well placed in the free falling elevator. Consider the operation "RESTORE TO "ONE", which leaves the particle in the "ONE" state, regardless of its initial location. If we assume that the particle is in the " 1 " state, then it is easy to leave it in the same state, without spending energy. If we are adopt that the particle is in the ZERO state, we can apply a force $\overrightarrow{\boldsymbol{f}}$ to it, which will push it over the barrier and transfer over the apex; and now, in a free falling elevator, this force may be infinitesimally small, and this is a great difference, from the original experiment, suggested in ref. 18. Afterwards we can apply a retarding force, so that when the particle arrives at ONE, it will have no excess kinetic energy, and we will not have expended any energy in the whole process, since we extracted energy from the particle in its downhill motion. Thus, at first sight it seems possible to RESTORE TO ONE without any expenditure of energy. This reasoning is true until thermal noise is neglected. If we assume that our particle $m$ is in a thermal equilibrium with isothermal surrounding $T$ thermal noise $c a . \boldsymbol{k}_{\boldsymbol{B}} \boldsymbol{T}$ should be surmounted in order to transfer the particle from the ZERO state to the ONE state. And what is extremely important: in a free falling elevator this results is independent on the mass $m$, due to the fact that effects to the gravity are eliminated. Thus, a large macroscopic body may be seen as a molecule $m$. Hence, if thermal equilibrium with surrounding is considered, the Landauer Principle remains true for the double-well system embedded into the free falling elevator.

We conclude, that in the absence of the dissipative processes (quantified by the thermal noise $\sim \boldsymbol{k}_{\boldsymbol{B}} \boldsymbol{T}$ states "ZERO" and "ONE" become indistinguishable, and just the thermal noise separates the aforementioned states. These arguments strengthen the fundamental role of the notion of "temperature" [28]; indeed, the appropriate choice of frames elimination of gravity; however, elimination of the thermal noise with the same procedure is impossible. Moreover the energy, necessary for the "RESTORE TO ONE" operation is independent on the mass of the particle, and depends only on the temperature of the system.

\subsection{The Einstein free falling elevator and the entropic forces}


Consider free jointed ideal polymer chain attached to the ceiling of elevator as shown in Figure 2. Particle $m$ is attached to the end of the chain. When the elevator is in rest the gravity force $\boldsymbol{m} \overrightarrow{\boldsymbol{g}}$ acting on the mass is balanced by the entropy elastic force $\overrightarrow{\boldsymbol{f}}_{\boldsymbol{e n}}$, supplied by Eq. 1 :

$$
\vec{f}_{e n}=\frac{3 k_{B} T}{N b^{2}} \vec{R}=m \vec{g},
$$

where $N$ and $b$ are the number and the length of the segment of free-joint chain correspondingly, and $\overrightarrow{\boldsymbol{R}}$ is the end-toend distance of the chain [29]. When the elevator is free falling, obviously $\overrightarrow{\boldsymbol{f}}_{\boldsymbol{e n}}=\mathbf{0}$ irrespectively of the mass $m$ connected to the chain. Now consider the free falling free-jointed chain and mass $m$ in the thermal equilibrium $T$ with the surrounding. The average force acting on the chain emerging from the random motion of the mass $m$ equals zero. Let us estimate the maximal entropic force emerging in the chain due to the thermal motion of the particle $m$ (we restrict ourselves by the one-dimensional random vertical motion of the particle). The general equation for the entropic force is:

$$
\left|\vec{f}_{e n}\right|=\left|T \frac{d S}{d y}\right|
$$

If the maximal vertical displacement of the chain is $\boldsymbol{\Delta} \boldsymbol{y}_{\max }$, we obtain:

$$
T \frac{\left|\Delta S_{\max }\right|}{\Delta y_{\max }} \Delta y_{\max } \cong k_{B} T
$$

where $\boldsymbol{\Delta} \boldsymbol{S}_{\max }$ is the maximal change in the entropy of the free-jointed chain due to the random thermal motion of the mass $m$. Eq. 2. Eq. 3 immediately yields:

$$
\left|\Delta S_{\text {max }}\right| \cong k_{B}
$$

And, again, it is immediately recognized from Eq. 4, that the maximal change in the entropy of the free-jointed chain attached to the free falling elevator in independent on the randomly walking mass $m$; moreover, it is independent on the structure of the free-jointed polymer chain (namely it is independent on the $N$ and $b$ ) and equilibrium temperature $T$. Absence of the dependence of $\boldsymbol{\Delta} \boldsymbol{S}_{\max }$ on the temperature of the chain is interesting in a view controversial nature of the relativistic transformation of temperature which remains highly debatable [30-31]. It is noteworthy, that the averaged random entropic force, emerging from thermal oscillations of the particle $m$ in the free falling elevator is zero; however, the entropic elastic force is not completely eliminated in the free falling frame, due to the fact that it emerges from the entropy of the chain and it is independent of gravity. Eq. 4 supplies re-interpretation of the Boltzmann constant, namely, the Boltzmann constant quantifies the maximal change in the entropy of the free-jointed polymer chain exposed to the thermal noise T. Consider that the role of gravity in our "Gedanken" experiment is "erased"; however, the role of the thermal noise is irremediable. 


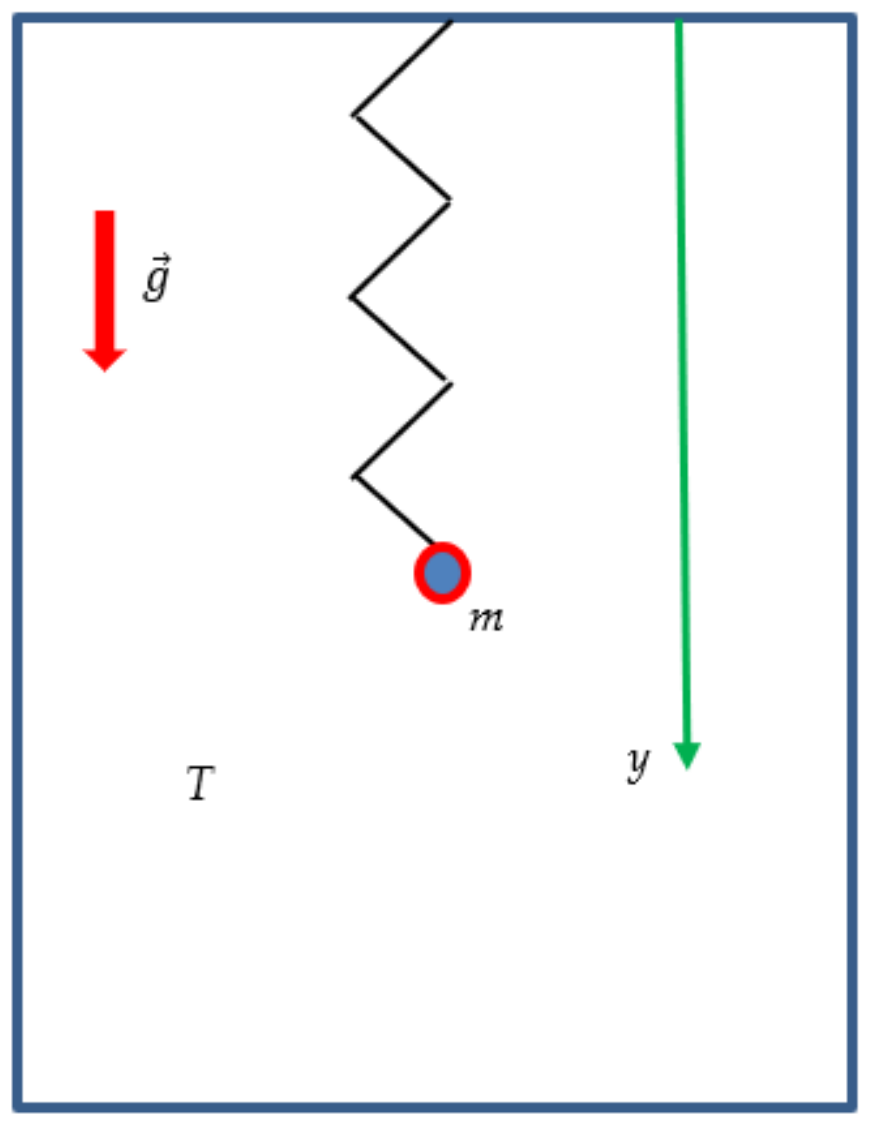

Figure 2. Free-falling elevator containing entropic spring (free-jointed polymer chain) and mass $m$ attached to the chain.

\subsection{Szilard minimal thermal engine in a free falling elevator}

Now consider the single-particle (minimal) thermal engine, suggested by Leo Szilard in 1929 [32, 33, 34]. This minimal thermal engine is based on a particle $\mathrm{m}$ enclosed within a chamber (cylinder) divided by half by a partition (piston) $\boldsymbol{M}_{2}$, as shown in Figures 3A-B. Szilard engine may be exploited for the exemplification of the Landauer Principle $[13,32,33]$. Finding of the particle $m$ in the certain (left or right) half of the chamber corresponds to the recording of 1 bit of information. When the partition is removed, the location of particle is uncertain, and this corresponds to the erasure of 1 bit of information. Location of a particle on the certain half of the chamber corresponds to "ONE", and the uncertain location of the particle corresponds to "ZERO", thus the single-particle-based engine provides the binary logical system $[13,32,33]$. The thermodynamic analysis of this computer-engine immediately gives rise to the Landauer bound; namely, the minimal energy necessary for isothermal erasing of one bit of information, which equals $\boldsymbol{k}_{\boldsymbol{B}} \boldsymbol{T} \boldsymbol{l n} \mathbf{2}$.

Let us place the minimal Szilard engine into the free falling Einstein elevator, as depicted in Figure 3. Various configurations of the Szilard engine shown in Figures 3A-B may be considered. Assume that the entire free falling systems shown in Figures 3A-B are in the thermal equilibrium with the surrounding at the temperature of $T$. Let to the particle $m$ perform isothermal expansion and push the piston $\boldsymbol{M}_{\mathbf{2}}$ under lifting up mass $\boldsymbol{M}_{\mathbf{1}}$. Kinetic energy of the particle $m$ will be spent for surmounting thermal noise $\sim \boldsymbol{k}_{\boldsymbol{B}} \boldsymbol{T}$ enabling lifting of the mass $\boldsymbol{M}_{\mathbf{1}}$ (the effects due to gravity are completely eliminated). The informational re-interpretation of the process sounds as follows: the energy $\boldsymbol{k}_{\boldsymbol{B}} \boldsymbol{T} \boldsymbol{l n} \mathbf{2}$ necessary for erasing of 1 bit of information was spent for lifting up mass $\boldsymbol{M}_{\mathbf{1}}$, whatever are the values of the masses $\boldsymbol{M}_{\mathbf{1}}$ and $\boldsymbol{M}_{\mathbf{2}}$. It is easily seen, that this conclusion is true for both vertical and horizontal configurations of the minimal 
Szilard engines, shown in Figures 2-3. Again, the appropriate choice of frames enables elimination of gravity; however elimination of the thermal noise (dissipation processes) by the same procedure is impossible.

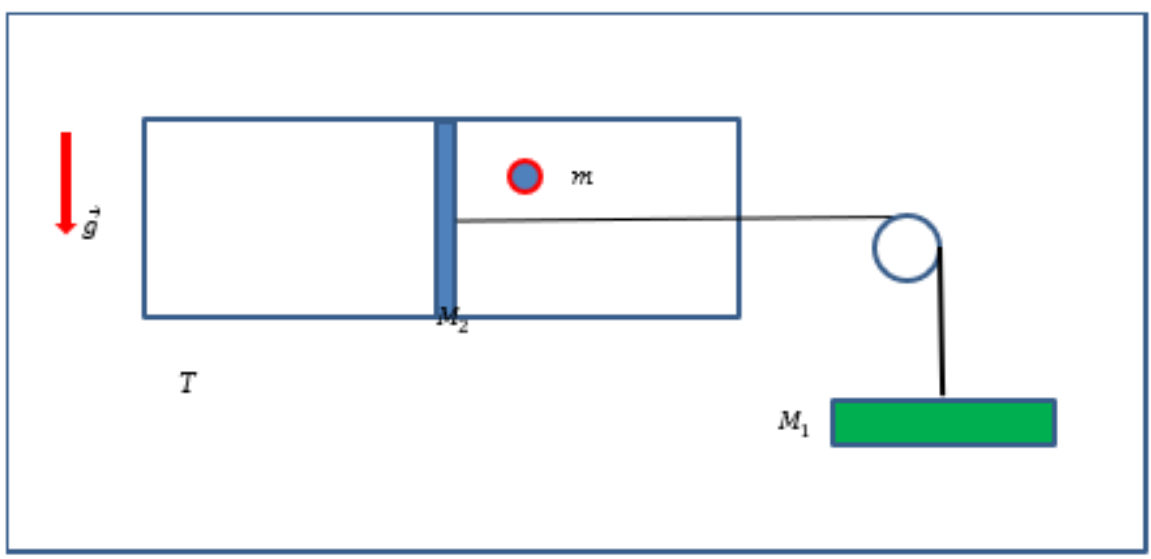

A

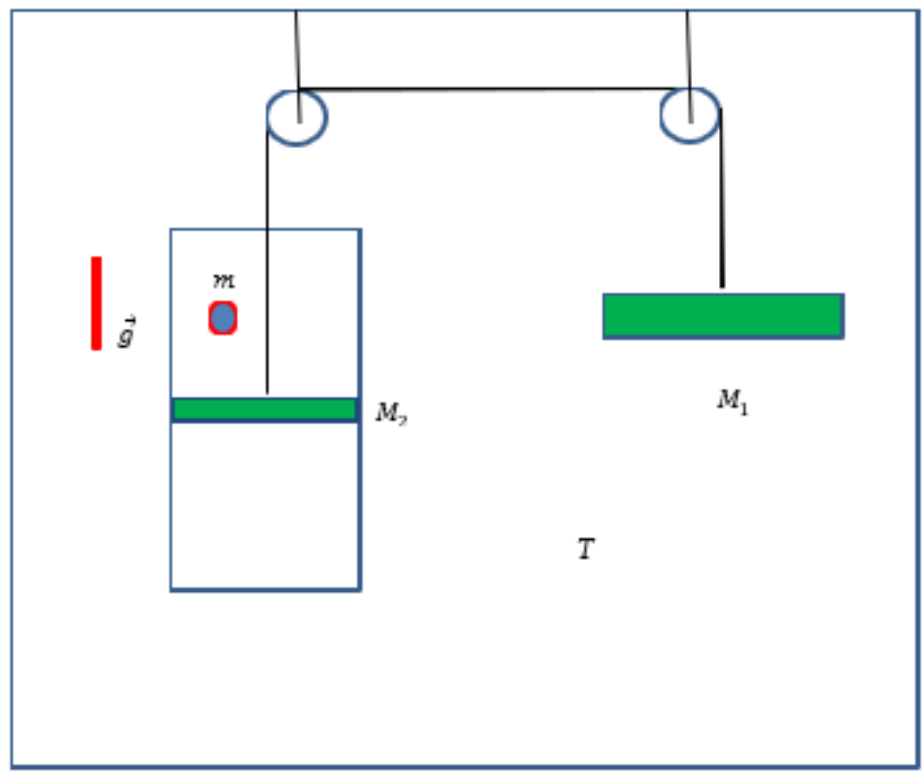

B

Figure 3A-B. Minimal single-particle thermal Szilard engines located in the free falling elevator.

\section{Conclusions}

We continue the development of the research program introduced by John Archibald Wheeler, suggesting that the basic notions of physics are deeply rooted in the theory of information [1]. We address the Gedanken experiments, combining the ideas emerging from the Einstein Principle of Equivalence [23-27] and the Landauer Principle [11-22]. For this purpose three experimental systems embedded into the free falling Einstein elevator are addressed, namely: the double well system containing particle $m$, the free jointed polymer chain with the mass $m$ attached to the chain, and the minimal Szilard engine driven by a single particle $m$. When the particle $m$ is confined within the free falling doublepotential-well system, representing the binary logics, it may be transferred from position "ZERO" to position "ONE", by an infinitesimal horizontal force. This is true until thermal noise is considered. Thus, the potential barrier of $\boldsymbol{k}_{\boldsymbol{B}} \boldsymbol{T}$ should be surmounted for the erasing of one bit of information in the suggested logical system. Ant it is important that this potential barrier is independent on the mass of the particle. Consider a free jointed polymer chain attached to the ceiling of free falling Einstein elevator. Mass $m$ is attached to the chain. The maximal change in the entropy of free-joint 
polymer chain attached to the free falling elevator is estimated as $\left|\boldsymbol{\Delta} \boldsymbol{S}_{\max }\right| \cong \boldsymbol{k}_{\boldsymbol{B}}$, and it is remarkably independent of the mass $m$ attached to the chain and the parameters of the chain, namely the number and length of the segments. Free falling minimal Szilard thermal engine, driven by the random motion of the single particle $m$ is treated [32-34]. The informational re-interpretation of the minimal Szilard process is formulated as follows: the energy necessary for erasing of 1 bit of information is spent for lifting up mass is on the order of magnitude of $\boldsymbol{k}_{\boldsymbol{B}} \boldsymbol{T}$, whatever is the value of this mass. We conclude, that the appropriate choice of frames enables elimination of gravity in a strict accordance to the equivalence principle; however elimination of the thermal noise (dissipation processes) by the same procedure is impossible for the addressed minimal informational systems.

\section{Author Contributions}

Conceptualization, E. B. and M. N.; Investigation, E. B. and M.N.; Writing and editing, E. B. and M. N. All authors have read and agreed to the published version of the manuscript.

\section{Funding}

The authors are thankful for funding to the Russian Science Foundation, Grant number 19-19-00076.

\section{Institutional Review Board Statement}

Not applicable.

\section{Informed Consent Statement}

Not applicable.

\section{Conflicts of Interest}

The authors declare no conflict of interest.

\section{References}

1. Wheeler, J.A. Information, physics, quantum: The search for links. In Proceedings of the III International Symposium on Foundations of Quantum Mechanics, Tokyo, Japan, 28-31 August 1989.

2. Ben Naim, A. Shannon's Measure of information and Boltzmann's H-Theorem. Entropy 2017, 19, 48.

3. Ben-Naim, A. Information Theory; World Scientific: Singapore, 2017.

4. Ben-Naim, A. A Farewell to Entropy: Statistical Thermodynamics Based on Information; World Scientific: Singapore, 2008.

5. Ben Naim, A. An Informational Theoretical Approach to the Entropy of Liquids and Solutions. Entropy 2018, 20, 514.

6. Ben-Naim, A. Entropy, the Truth the Whole Truth and Nothing but the Truth; World Scientific: Singapore, 2016.

7. Müller, J. G Information Contained in Molecular Motion. Entropy 2019, 21(11), 1052.

8. Knuth, K.H. Information-Based Physics and the Influence Network. In It from Bit or Bit from It? The Frontiers Collection; Aguirre, A., Foster, B., Merali, Z., Eds.; Springer: New York, NY, USA, 2015.

9. Knuth, K.H.; Walsh, J.L. An Introduction to Influence Theory: Kinematics and Dynamics. Ann. Phys. 2018, 531, 1800091.

10. Caticha, A. Entropic Dynamics. Entropy 2015, 17, 6110-6128.

11. Vopson, M.M. The mass-energy-information equivalence principle. AIP Adv. 2019, 9, 095206.

12. Müller, J. G. Observable and Unobservable Mechanical Motion, Entropy 2020, 22(7), 737.

13. Bormashenko, Ed. Informational Reinterpretation of the Mechanics Notions and Laws. Entropy 2020, $22(6), 631$.

14. Bormashenko, Ed. The Landauer Principle: Re-Formulation of the Second Thermodynamics Law or a Step to Great Unification? Entropy 2019, 21(10), 918.

15. Müller, J. G. Photon Detection as a Process of Information Gain. Entropy 2020, 22(4), 392.

16. Herrera, L. Maxwell Demon's and the Problem of Observers in General Relativity. Entropy 2018, $20,391$. 
17. Herrera, L. Landauer Principle and General Relativity. Entropy 2020, 22(3), 340.

18. Landauer, R. Irreversibility and heat generation in the computing process. IBM J. Res. Dev. 1961, 5(3), $183-191$.

19. Landauer, R. Information is physical. Phys. Today 1991, 44, 23-29.

20. Landauer, R. Minimal energy requirements in communication. Science 1996, 272, 1914-1918.

21. Norton, J.D. Waiting for Landauer. Stud. Hist. Philos. Sci. B 2011, 42, 184-198.

22. Bennett Ch. H. Notes on Landauer's principle, Reversible Computation and Maxwell's Demon, Studies History E Philosophy Modern Phys. 2003, 34 (3), 501-510.

23. Landau, L.; Lifshitz, E.M. The Classical Theory of Fields, 4th ed.; Butterworth-Heinemann: Oxford, UK, 1975 ; Volume 2.

24. Tolman, R.C. Relativity, Thermodynamics and Cosmology; Oxford University Press: Oxford, UK, 1934.

25. Earman, J.; Friedman, M. The meaning and status of Newton's Law of inertia and the nature of gravitational forces. Philos. Sci. 1973, 4, 329-359.

26. Roll, P.G.; Krotkov, R.; Dicke, R.H. The equivalence of inertial and passive gravitational mass. Ann. Phys. 1964, 26, 442517.

27. Ohanian, H. What is the principle of equivalence? Am. J. Phys. 1977, 45, 903.

28. Bormashenko, Ed. What Is Temperature? Modern Outlook on the Concept of Temperature, Entropy 2020, 22 ( 12 ), 1366.

29. Rubinstein, M.; Colby R. H. Polymer Physics, Oxford Press, NY, USA, 2003.

30. Landsberg, P.; Matsas, G.E.A. The impossibility of a universal relativistic temperature transformation. Phys. A Stat. Mech. Appl. 2004, 340, 92-94.

31. Bormashenko, E. Entropy of Relativistic Mono-Atomic Gas and Temperature Relativistic Transformation in Thermodynamics. Entropy 2007, 9, 113-117.

32. Szilard, L. Über die Entropieverminderung in einem thermodynamischen System bei Eingriffen intelligenter Wesen. J. Phys. 1929, 53, 840-856. (In German).

33. Lutz, E.; Ciliberto, S. Information: From Maxwell's demon to Landauer's eraser. Phys. Today 2015, 68, 30-35.

34. Bormashenko, E.; Shkorbatov, A.; Gendeman, O. The Carnot engine based on the small thermodynamic system: Its efficiency and the ergodic hypothesis. Am. J. Phys. 2007, 75, 911-915. 